\title{
Subsurface imaging of silicon nanowires circuits and iron oxide nanoparticles with sub-10 nm spatial resolution
}

\author{
A.P. Perrino, Y.K. Ryu, C. A. Amo, M. P. Morales, R. Garcia* \\ Instituto de Ciencia de Materiales de Madrid, CSIC \\ c/ Sor Juna Ines de la Cruz 3, 28049 Madrid, Spain. \\ *email address: r.garcia@csic.es
}

\begin{abstract}
Non-destructive subsurface characterization of nanoscale structures and devices is of significant interest in nanolithography and nanomanufacturing. In those areas, the accurate location of the buried structures and their nanomechanical properties are relevant to optimize the nanofabrication process and the functionality of the system. Here we demonstrate the capabilities of bimodal and trimodal force microscopy to image silicon nanowire devices buried under an ultrathin polymer film. We resolve the morphology and periodicities of silicon nanowire pairs. We report a spatial resolution in the sub-10 nm range for nanostructures buried under a $70 \mathrm{~nm}$ thick polymer film. By using numerical simulations we explain the roles of the excited modes in the subsurface imaging process. Independently of the bimodal or trimodal AFM approach, the fundamental mode is the most suitable to track the topography while the higher modes modulate the interaction of the tip with the buried nanostructures and provide the subsurface contrast.
\end{abstract}




\section{Introduction}

Non-destructive characterization of buried or internal nanometer-scale structures has a wide range of implications in different fields from cell biology[1,2], toxicity[3] polymer sciences[4,5] or semiconductor device fabrication[6]. A variety of methods ranging from confocal, transmission electron[3] or near-field microscopies have been proposed[7] to image subsurface structures. Ultrasound waves and force microscopy configurations [8] have been proposed to investigate embedded structures [9-17]. Electrostatic and Kelvin probe force microscopy have been applied to reveal the structure of buried polymer nanocomposites [18,19]. Anomalies in the flow-shift paradigm of spin-coated films at the submicrometer scale have been exploited for precise overlay fabrication in thermal scanning probe lithography[20]. However, the spatial resolution of the above methods is in the submicrometer range. The long-range character of the electrostatic interactions in bias-based probe methods or the wavelength of ultrasound waves in acoustic-based force microscopy limits the spatial resolution to the 50-100 $\mathrm{nm}$ range.

Recently, Solares and co-workers have reported a multifrequency AFM subsurface imaging method[21]. They developed a trimodal AFM approach[21-23] to image glass nanoparticles buried under a polymer film. This approach involves the simultaneous excitation and detection of three eigenmodes to measure the topography, modulate the sample indentation and map compositional contrast. This multifrequency AFM approach has opened a novel and potentially high spatial resolution method for subsurface characterization. However, many key aspects of this approach have not been addressed. Are three modes needed to achieve subsurface imaging? Could the same effect be achieved with monomodal or bimodal excitation and detection? What properties should have a flexural mode to enable subsurface contrast? Only a single system, glass nanoparticles have been imaged by multifrequency AFM. Additional examples are needed to establish the general character of the method.

Here, we demonstrate the capability of both bimodal and trimodal AFM to image silicon nanowire (SiNWs) arrays and iron oxide nanoparticles (NPs) buried under a spin-coated film. The images show a lateral resolution in the sub-10 $\mathrm{nm}$ range. Furthermore, we provide some examples that illustrate that the subsurface imaging process preserves the spatial resolution of tapping mode AFM operation. We have developed a theoretical framework to understand the contrast mechanisms and explain the different roles of the excited modes in the subsurface imaging process. We show that both bimodal and trimodal configurations are suitable for subsurface imaging. The first mode provides the topography mapping. The second mode enhances the contrast of the buried nanostructures and facilitates the interaction with the nanostructures. If a third mode is used, its role is to favor the tip interaction with the buried nanostructures.

\section{Experimental methods}

\subsection{Silicon Substrates}


Silicon substrates have been cleaned with 2-propanol, acetone and distilled water (SigmaAldrich) by ultrasonic treatment for 5 minutes each. The substrates were then immersed in a $\mathrm{H}_{2} \mathrm{O}_{2}-\mathrm{NH}_{4} \mathrm{OH}-\mathrm{H}_{2} \mathrm{O}(1: 1: 2)$ mixture and four ultrasound cycles of 10 minutes have been performed.

\subsection{Fabrication of silicon nanowires by oxidation SPL}

Silicon on insulator substrates with a Si active layer $12 \mathrm{~nm}$ thick, p-doped and a nominal resistivity of 9-15 $\Omega \mathrm{cm}$ (MEMC/SunEdison, US) and a buried oxide layer (BOX) $25 \mathrm{~nm}$ thick were used for the fabrication of the devices. The substrates were firstly cleaned with a $\mathrm{NH}_{4} \mathrm{OH}-$ $\mathrm{H}_{2} \mathrm{O}_{2}-\mathrm{H}_{2} \mathrm{O}(1: 1: 4)$ mixture in three ultrasound cycles of 10 minutes and a last ultrasound cycle of 5 minutes in deionized water. Then Ti/Au marker electrodes were defined by photolithography to localize the structures. An interdigitated array of silicon nanowires were fabricated after definition of $\mathrm{SiO}_{2}$ masks by oxidation scanning probe lithography and pattern transfer by dry etching processing. The o-SPL was performed by operating the AFM (Dimension

$\mathrm{V}$, Bruker, USA) in the amplitude modulated mode with a free amplitude of $5 \mathrm{~nm}$ and a set point amplitude/free amplitude ratio of about 0.9. n+-doped silicon cantilevers (NCH-W, NanoWorld) with a force constant of about $40 \mathrm{~N} / \mathrm{m}$ and a resonant frequency about $300 \mathrm{kHz}$ were used for the fabrication of the patterns. The relative humidity was kept at about $45 \%$ in a sealed chamber. Voltage pulses of 22.5-24 V and $1 \mathrm{~ms}$ were used. The silicon oxide mask thickness and width are, respectively, in the range of 1.5-2.2 nm and 40-70 nm. After reactive ion etching (NRE3000, INNOVA Scientific) process, using a gas mixture proportion, chamber pressure, radiofrequency power and etching time of, respectively, $\mathrm{SF}_{6}: \mathrm{O}_{2}(10: 5) \mathrm{sccm}, 59 \mathrm{mTorr}, 15 \mathrm{~W}$ and $43 \mathrm{~s}$, silicon nanowires which preserve the original thickness of the top Si layer were produced.

\subsection{Nanoparticles deposition}

In order to deposit the $\mathrm{Fe}_{2} \mathrm{O}_{3}$ nanoparticles, the silicon surface was functionalized after the cleaning procedure. The substrates were immersed in a solution containing $11 \mu 13$-aminopropyltriethoxysilane (APTES) and $50 \mathrm{~mL}$ ethanol for 45 minutes. Finally, the substrates were rinsed with ethanol and water, and dried under $\mathrm{N}_{2}$. Afterwards, the silicon surface was covered with the dimercaptosuccinic acid (DMSA) coated $\mathrm{Fe}_{2} \mathrm{O}_{3}$ nanoparticles by the drop casting method. A 20 $\mu \mathrm{l}$ drop taken from a $1.4 \mathrm{mg} / \mathrm{ml}$ nanoparticles aqueous solution was deposited on the silicon surface for 60 seconds.

\subsection{Polydimethylsiloxane (PDMS) substrates}

The chips containing the silicon nanowires and the nanoparticles were spin coated by a mixture of PDMS (Sylgard 184, Sigma Aldrich) curing agent: PDMS elastomer base: hexane (Scharlau, Scharlab, S.L.) with a proportion of 1:10:1000 (by weight) at $6000 \mathrm{rpm}$ for $180 \mathrm{~s}$ and then cured 
on a hot plate at $150{ }^{\circ} \mathrm{C}$ for 10 minutes. Under these conditions, the PDMS films have thicknesses between $30 \mathrm{~nm}-60 \mathrm{~nm}$.

\subsection{AFM Measurements}

The experiments have been performed with a Cypher S microscope (Asylum Research, Santa Barbara, USA). We have used PPP-FMAuD cantilevers (Nanosensors) with typical values of $k_{1}$ $\approx 2.3 \mathrm{~N} / \mathrm{m}, f_{01} \approx 66 \mathrm{kHz}$ and $Q_{1} \approx 190 Q_{1} \approx 190$. The cantilever has been driven at the three first eigenmodes simultaneously in the trimodal AM scheme[24]. The amplitudes of the modes have been adjusted to achieve compositional contrast, stable imaging and the indentation needed for the subsurface imaging process.

\subsection{Multifrequency AFM theory and simulations}

The solution of the modified Euler-Bernouilli beam equation for a uniform, continuous and rectangular microcantilever is approximated by a system of three point-mass equations coupled by the force term [25],

$$
m \frac{d^{2} z_{i}(t)}{d t_{i}^{2}}+\frac{m \omega_{0 i}}{Q_{i}} \frac{d z_{i}(t)}{d t_{i}}+k_{i} z_{i}(t)=\sum_{j=1}^{3} F_{0 j} \cos \omega_{j} t+F_{t s}\left(z_{c}+z_{1}(t)+z_{2}(t)+z_{3}(t)\right) i=1,2,3
$$

where $m$ is the reduced mass of the cantilever and $z_{i}, \mathrm{Q}_{\mathrm{i}}, f_{i}, \omega_{i}=2 \pi f_{i}, \omega_{0 i}=2 \pi f_{0 i}, k_{i}, A_{0 i}$ and $F_{0 i}$ are respectively the deflection, quality factor, driving frequency, angular driving frequency, resonant frequency, angular resonant frequency, stiffness, free amplitude and driving force amplitude of the $i$-th flexural mode.

$F_{t s}$ is the tip - sample force and $z_{c}$ is the cantilever - sample distance. The flexural modes parameters are $f_{1}=f_{01}=66.7 \mathrm{kHz}, f_{2}=f_{02}=421 \mathrm{kHz}, f_{3}=f_{03}=1.18 \mathrm{MHz}, k_{1}=2.3 \mathrm{~N} / \mathrm{m}, k_{2}=$ $88.15 \mathrm{~N} / \mathrm{m}, k_{3}=800 \mathrm{~N} / \mathrm{m}, Q_{1}=173, Q_{2}=400, Q_{3}=771$. The equations of motion were integrated numerically using a fourth order Runge-Kutta algorithm. . The solutions of (1) are given approximately by

$$
z_{i}\left(z_{c}, t\right)=A_{i}\left(z_{c}\right) \cos \left(\omega_{i} t-\phi_{i}\left(z_{c}\right)\right)
$$

where $A_{i}, \phi_{i}$ are the amplitude and phase shift of the i-th flexural mode. The tip-sample interactions are described by the Van der Waals interaction for the attractive regime and by the Derjaguin-Muller-Toporov and Kelvin - Voigt model as is described in [26]

$$
F_{t s}(d)=\left\{\begin{array}{cc}
-H R_{t} / 6 d^{2} & d \geq a_{0} \\
4 / 3 E_{f f} \sqrt{R_{t} \delta^{3}}-H R_{t} / 6 a_{0}^{2}-\eta \sqrt{R_{t} \delta}(d \delta / d t) & d \leq a_{0}
\end{array}\right.
$$


where $d \equiv d(t)=z_{c}+z_{1}(t)+z_{2}(t)+z_{3}(t)$ is the instantaneous tip - sample distance, $a_{0}=0.165$ $\mathrm{nm}$ is the intermolecular distance, $\delta(t)$ is the indentation, $\eta=5 \mathrm{~Pa}$ is the dynamic viscous coefficient $R_{t}$ is the tip radius,

$$
E_{f f}=\left(\frac{1-v_{t}^{2}}{E_{t}}+\frac{1-v_{s}^{2}}{E_{s}}\right)^{-1}
$$

is the effective Young modulus and $v_{x}, E_{x}$ are the sample $(s)$ and tip $(t)$ Poisson's ratios and Young modulus respectively. We use $v_{s}=v_{t}=0.3, E_{s}=10 \mathrm{MPa}, E_{t}=170 \mathrm{GPa}$. We consider a spherical tip of negligible mass $\left(R_{t}=10 \mathrm{~nm}\right)$ only for interaction purposes.

\section{Results and discussion}

\subsection{Multifrequency AFM: Bimodal or trimodal excitation/detection schemes}

The multifrequency AFM approach for subsurface imaging involves the simultaneous excitation of several cantilever modes, either two (bimodal) or three (trimodal). Bimodal AFM is the most robust multifrequency-based probe approach for topography and quantitative mechanical characterization of surfaces at the nanoscale. The fundamentals of bimodal AFM and its extensions are described in several reviews[24][27,28] and research contributions[29-37].

Subsurface imaging in a trimodal configuration is more complex than in bimodal AFM, for this reason, trimodal AFM operation for subsurface imaging is depicted in Figure 1(a). The first three flexural modes of the microcantilever are excited by a signal that contains three sinusoidal components. The components are tuned, respectively, to the first, second and third cantilever flexural modes. In the optimum operating conditions, the amplitudes of the components are asymmetric, the largest amplitude value corresponds to the $1^{\text {st }}$ mode $A_{1}$, next is $A_{3}$ and then $A_{2}$. This scheme enhances the indentation of the polymer layer $\left(A_{3}>A_{2}\right)$ and improves compositional contrast. In the case of bimodal AFM $A_{1}$ is larger than $A_{2}$.

Figure 1b-c illustrate the type of nanostructures investigated to demonstrate the subsurface imaging capabilities. The topography (Fig. 1(d)) and thickness (Fig. 1(e)) of the spin-coated film are shown. For example, the cross-section across the edge of a spin-coated silicon region reveals a thickness of $70 \mathrm{~nm}$ (Fig. 1(e)). The thickness values that are directly extracted from the AFM data are smaller $(\sim 45 \mathrm{~nm})$. The force applied during the imaging process introduces some deformation on the polymer[38,39]. The true profile (continuous line) is reconstructed by using a numerical simulation code [26]. The topography peak observed at the edge of the coated/uncoated region is due to the scratching process applied to generate coated and uncoated regions on the Si substrate. 


\subsection{Silicon nanowire and iron oxide nanoparticles buried under a polymer film}

Two different nanoscale systems have been chosen to test multifrequency AFM for subsurface imaging, silicon nanowires and nanoparticles. Figure 2(a) shows an optical image of the gold contacts of a silicon nanowire circuit. The square indicates the region that contains the SiNW array. The amplitude modulated (tapping mode) AFM [40] image (Fig. 2(b)) shows a set of interdigitated SiNWs fabricated by oxidation scanning probe lithography (o-SPL)[41,42]. The SiNWs are $2 \mu \mathrm{m}$ in length, $40 \mathrm{~nm}$ in width (average value at half maximum). The separation between nanowires ranges between 49 and $98 \mathrm{~nm}$. To demonstrate the capability to sub-10 nm particles, we have deposited iron oxide nanoparticles $[43,44]$ on a $\mathrm{Si}(100)$ surface (Fig. 2(c)). The nanoparticles have a diameter distribution between 7 and $10 \mathrm{~nm}$ with an average diameter centered at $8.2 \mathrm{~nm}$ (Fig. 2(d)).

To illustrate that both the amplitude and the phase shift observables in multifrequency AFM are sensitive to subsurface features we plot the topography and phase shift contrast $\left(\Delta \phi_{1}\right)$ images of an array of SiNWs and a random distribution of NPs after they have been buried under an approximately $70 \mathrm{~nm}$ layer of polydimethylsiloxane (PDMS). Both observables provide good contrast of the spin-coated SiNWs (Fig. 3(a), (b)) and iron oxide NPs (Fig. 3(c), (d)).

The capabilities of multifrequency AFM to reveal the morphology of nanostructures buried under polymer films are demonstrated by comparing the images obtained, respectively, by tapping mode and trimodal AFM of the same region of the sample. Figure $4 \mathrm{a}$ and $4 \mathrm{~b}$ show, respectively, the trimodal and the tapping mode AFM images of the same SiNW circuit. The SiNWs are only resolved in the trimodal AFM image. Identical results are obtained by performing the comparison with the buried NPs (Fig. 4(c) and 4(d)). The above results are independent of the imaging acquisition sequence (first tapping mode then trimodal or first trimodal then tapping mode).

The cross-sections along the marked lines shown in Fig. 2(b) and Fig. 4 illustrate the genuine character of the subsurface contrast of multifrequency AFM (Fig. 5(a)-(b)). The tapping mode AFM cross-section of an array of SiNWs coated by PDMS does not provide any hint on the presence of the nanostructures. However, the buried nanowires are resolved if the AFM configuration is switched to trimodal AFM. Furthermore, the trimodal AFM profile matches the one obtained by tapping mode AFM before the deposition of the PDMS film. We observe that the subsurface cross-section is slightly sharper than the cross-section of the same nanowires before PDMS deposition. This could be attributed to a change of the tip geometry. It also points out that the subsurface imaging process does not necessarily imply less spatial resolution. Similar result is obtained with the NPs (Fig. 5(b)).

In the above comparison, we have used the same cantilever for tapping and trimodal AFM modes. The free amplitude $A_{0}$ was higher in tapping mode AFM $\left(A_{0}=160 \mathrm{~nm}\right)$ than in trimodal 
AFM (total amplitude $\sim 142 \mathrm{~nm}$ ). This demonstrates that the operational amplitudes for imaging are not the dominant parameters in the subsurface imaging contrast.

\subsection{Simulations of trimodal AFM imaging}

We have performed numerical simulations of the cantilever dynamics under the simultaneous excitation of the first three flexural modes. The simulations are intended to clarify the contrast mechanism and to explain the role of the different eigenmodes in the subsurface imaging process. It has been shown that the vertical resolution $\Delta h$ (the minimum step height variation that can be measured) is inversely proportional to the slope of the amplitude curve $\left(d A / d z_{c}\right)$ [45],

$$
\Delta h=\frac{\Delta A}{\left|d A / d z_{c}\right|}
$$

where $\Delta A$ is the error in the amplitude $A$ and $z_{c}$ is the probe height. The maximum possible value of the above slope is 1 . Equation 5 shows that the vertical resolution increases by decreasing the slope. At the same time, slopes below 1 will diminish the ability of the AFM to track the surface topography.

Figure 6(a) shows the dependence of the amplitude with respect to the tip-sample separation (probe height) for different monomodal amplitude modulation AFM (AM-AFM) configurations. The slope in the central region decreases with the mode order from 1 ( $1^{\text {st }}$ mode $)$ to $0.4\left(2^{\text {nd }}\right.$ mode $)$ and $0.02\left(3^{\text {rd }}\right.$ mode). Definitely a feedback set on $A_{3}$ will have an extremely poor microscopy performance. The second mode is still suitable for imaging flat surfaces but unsuitable to track surfaces with features of tens or hundreds of nanometers in height. This result is related to the sensitivity of the cantilever modes which for the phase shift scales with [27] [45],

$$
\Delta \phi_{i}=K \frac{Q_{i}}{k_{i}}
$$

where $K$ is a factor that depends on the type of interaction force acting on the cantilever and $Q_{i}$ and $k_{i}$ are, respectively, the quality factor and force constant of the $i$-th flexural mode of the microcantilever.

In multifrequency AFM the slope $d A / d z_{c}$ shows a sharp dependence on the mode order. Figure $6 \mathrm{~b}$ shows the slope corresponding to $A_{1}$ for different monomodal, bimodal and trimodal configurations. In all the configurations the slope in the central region is approximately 1.

Consequently, $A_{1}$ is a suitable observable to track the topography. A different result is obtained in multifrequency AFM when the slopes corresponding to $A_{2}$ and $A_{3}$ are plotted (Fig. 6(c)). The $d A_{i} / d z_{c}$ values $(i=2,3)$ are close to zero. 
We have compared the experimental and theoretical dependence of the amplitude curves on the excited mode for monomodal excitations. Figure 7(a) shows some experimental amplitude curves obtained on a PDMS sample. The curves show that the slope decreases with the excited mode. The first mode has a slope close to 1 while for the second and third modes the slopes are, respectively, 0.5 and 0.05 . The simulations shown in Fig. 7(b) confirm the experimental observation by reproducing the trend described above. In addition, the slope values reported for the different modes are close to the experimental values.

A critical aspect in the subsurface imaging process is the tip's ability to interact with the nanostructures buried under the polymer film. We have studied the tip penetration as a function of the AFM configurations. Figure 8(a) shows that in monomodal excitation and detection the tip penetration increases with the mode order. For $z_{c}=60 \mathrm{~nm}$, the penetration is $23.5 \mathrm{~nm}$ for the $1^{\text {st }}$ mode, $70 \mathrm{~nm}$ for the $2^{\text {nd }}$ mode and $78 \mathrm{~nm}$ for the $3^{\text {rd }}$ mode. This indicates that for the same operational conditions, the penetration of the first mode is significantly smaller than that of the $2^{\text {nd }}$ and $3^{\text {rd }}$ modes. This could be explained in terms of the maximum potential energy of the microcantilever. The energy is proportional to the $k_{i}$ which grows with the resonant frequency of the excited mode $\left(f_{0 i}\right)$. For a rectangular cantilever [27],

$$
k_{i}=k_{1}\left(\frac{f_{0 i}}{f_{01}}\right)^{2}
$$

Consequently, for the same set-point amplitude the maximum potential energy stored in the microcantilever grows with the mode order.

A different result is obtained in multifrequency AFM. At $z_{c}=60 \mathrm{~nm}$, the penetration is about 32 $\mathrm{nm}$ for the three configurations studied here (Fig. 8(b)). That value is in between the value calculated for the tip penetration of the $1^{\text {st }}$ mode and that of the $2^{\text {nd }}$ and $3^{\text {rd }}$ modes.

The phase shift of the $2^{\text {nd }}$ mode is very sensitive to the compositional properties of the material $[25,46]$. Figure 9 compares the phase shift contrast $\Delta \phi$ obtained between a region of $10 \mathrm{MPa}$ and another region of $1 \mathrm{GPa}$ for different multifrequency AFM configurations. In bimodal AFM, the configuration with the smallest contrast is obtained in $\Delta \phi_{3}$ by exciting the $1^{\text {st }}$ and the $3^{\text {rd }}$ modes (Fig. 9(a)). For trimodal AFM, the contrast is maximized by using the phase shift of the $2^{\text {nd }}$ mode. Nonetheless, it should be emphasized that in both cases the phase shift contrast is well above the noise level.

Imaging soft matter with the AFM always involves some sort of sample deformation. This property has enabled the use of force microscopy to study the nanomechanical properties of tissues[2] and cells [47,48]. Here, the deformation is exploited to detect the nanostructrures buried under a thin polymer film. The existence of deformations is independent of the single frequency or the multifrequency character of the probe excitation and detection schemes, 
however, bimodal or trimodal AFM optimizes the indentation and the subsurface imaging contrast versus standard (single frequency) AFM methods.

The tip's potential energy depends on the force constant and the amplitude. For a rectangular cantilever the force contact ratio grows with the square of the resonant frequency that would favour the use of higher modes for penetration. However, the sensitivity of higher modes decreases with the mode order [49] (see Fig. 6) so the ability to properly track the topography is reduced. On the other hand, the slope of the first mode hardly depends on the AFM configuration (single mode, bimodal or trimodal). This implies that the ability $A_{1}$ to track the sample topography remains unaffected in multifrequency AFM. Then the introduction of an additional excitation will open additional channels and increase the tip indentation (Fig. 8).

The above considerations have been supported by the numerical simulations. The simulations confirm that the feedback to track the sample topography must be connected to the $A_{1}$. The slope $A_{1}$ with the probe height is the highest (Fig. 6(a)), which guarantees the optimum conditions for topography imaging. Second, the additional excitation of one or two higher modes enhances the tip penetration. This property enables the tip to interact with the buried nanostructures. It has been established that the material properties contrast depends on the observable. An enhanced phase contrast is found by plotting the phase shift of the $3{ }^{\text {rd }}$ mode. The above factors explain the capabilities of trimodal AFM to perform subsurface imaging. The data also illustrates that the spatial resolution in trimodal AFM is similar to the one obtained with the SiNWs before being coated by the PDMS layer.

Figure 8 illustrates that trimodal excitation is not strictly needed for achieving subsurface imaging. The slope of the $A_{1}$ curve in bimodal AFM $(130,12,0)$ and $(130,0,12)$ is almost identical to the slope of the $A_{1}$ curve in a trimodal configuration $(130,2,10)$. The compositional contrast is smaller $\left(\Delta \phi_{\text {bimodal }} \approx 15^{\circ}\right.$ versus $\left.\Delta \phi_{\text {trimodal }} \approx 40^{\circ}\right)$. However, in both configurations $\Delta \phi$ is well above the noise level $\left(\sim 0.5^{\circ}\right)$. In practice, this means that both bimodal or trimodal AFM are suitable to perform subsurface imaging.

The similar dependence of the tip's indentation on the probe height (Fig. 8(b)) emphasizes a property of multifrequency AFM operation. It reflects that in multifrequency AFM, the amplitude reduction of the higher modes is not controlled by the $Q_{i} / k_{i}$ ratio (Eq. 6 ) provided that the amplitudes $\left(A_{2}, A_{3}\right)$ are smaller than $A_{1}$. The energy transfer from the first mode to the higher excited modes is a factor which compensates the amplitude reduction due to the tip-surface forces that happens in monomodal excitation and detection (Fig. 6(a)).

\section{Conclusions}

We have studied and demonstrated the capability of bimodal and trimodal AFM to image nanostructures buried under thin polymer films. Silicon nanowires of $12 \mathrm{~nm}$ in height and about $40 \mathrm{~nm}$ in diameter buried under a $70 \mathrm{~nm}$ soft polymer film (1-10 MPa) have been imaged. Iron 
oxide nanoparticles with a mean diameter of $8.2 \mathrm{~nm}$ have also been detected. By combining theory and numerical simulations we have explained the role of the different excited modes in the subsurface imaging process. The amplitude of first mode is the optimum observable to track the sample topography of the external interface while the higher excited modes contribute to enhance the subsurface contrast or facilitate the sample deformation. In bimodal AFM, the signal of the second mode has a dual role, it provides the tip-subsurface structure interaction and the subsurface contrast. Trimodal AFM assigns specific roles to the three excited modes. The first mode carries the topography contrast, the second mode optimizes the compositional contrast and the third mode modulates the tip penetration. Trimodal AFM operation offers a higher subsurface contrast than bimodal AFM.

The success of multifrequency AFM to image buried nanostructures depends on the softness of the interface. This method will not be suitable to inspect embedded nanostructures in a rigid matrix, say with a Young modulus above $500 \mathrm{MPa}$. However, there is a variety of applications in nanofabrication and nanolithography where soft resists layers are used. The inspection of those samples before further processing represents an application niche for subsurface imaging by multifrequency AFM. In cell biology and nanotoxicity studies, the cell's uptake of nanoparticles and nanotubes, and its location respect to cell organelles could also be examined by this method.

\section{Acknowledgments}

This work was funded by the European Union FP7/2007-2013 under Grant Agreement No. 318804 (SNM), the Ministerio de Economía y Competitividad (Spain) under grants MAT201344858-R, CSD2010-00024 and the European Research Council ERC-AdG-340177 (3DNanoMech). 
Figures

(a)
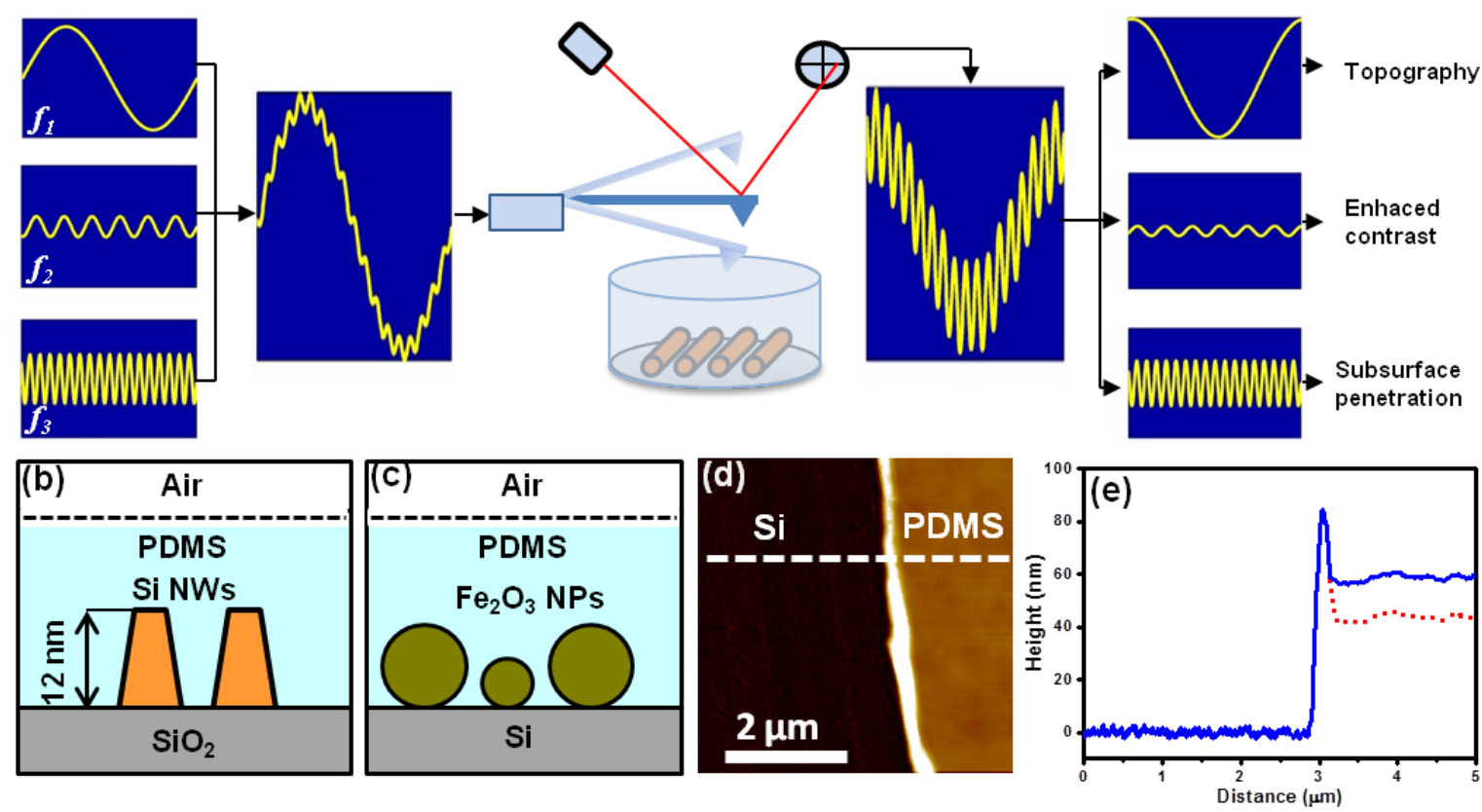

Figure 1. (a) Scheme of trimodal AFM for subsurface imaging. The driving force of the cantilever involves three components tuned at the first, second and third flexural modes of the cantilever beam. The $1^{\text {st }}$ mode tracks the topography of the sample; the $2^{\text {nd }}$ mode provides subsurface contrast and the $3^{\text {rd }}$ mode modulates the subsurface penetration. (b) Scheme of the silicon nanowires buried under a spin-coated PDMS film. (c) Scheme of the iron oxide nanoparticles buried under a spin-coated PDMS film. (d) Amplitude modulation AFM (tapping mode) image of a region with and without the PDMS film; $A_{s p}=120 \mathrm{~nm} ; A_{0}=200 \mathrm{~nm}$. (e) Cross-section along the line marked in panel (d). The profile reveals the thickness of the film (continuous line). The dash line represents the unreconstructed height profile. The peak at the edge is an artifact generated during the scratching process to generate a coated/uncoated interface. 

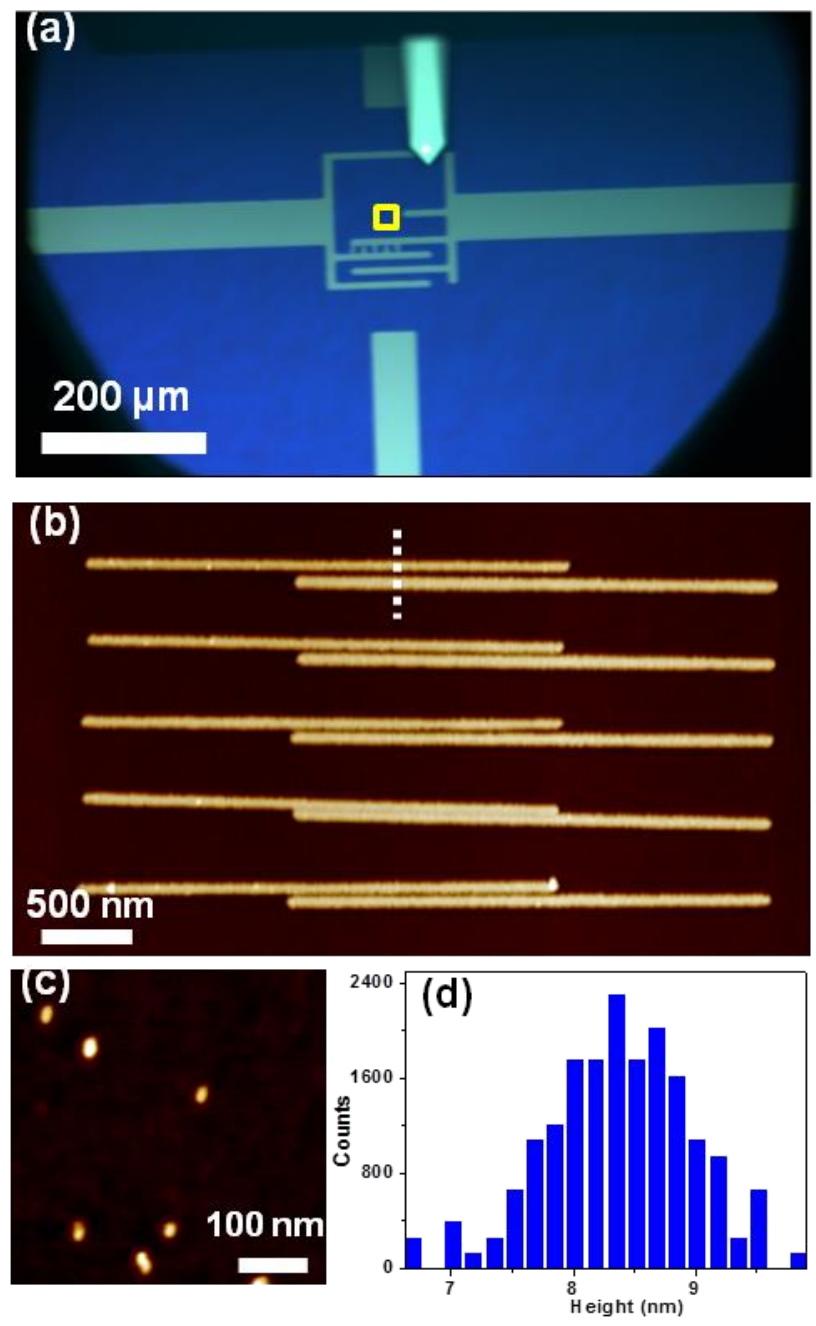

Figure 2. (a) Optical image of the metallic contacts of a silicon nanowire circuit. The marked square indicates the region that contains the SiNWs. The AFM cantilever is also seen in the image. (b) Tapping mode (amplitude modulation) AFM image of an array of SiNWs that have been fabricated by oxidation SPL in the marked region. $A_{s p}=4.5 \mathrm{~nm} ; A_{0}=5 \mathrm{~nm}$. The image shows the array before the spin-coated process. (c) Tapping mode AFM image of NPs deposited on a Si surface. $A_{s p}=6.5 \mathrm{~nm} ; A_{0}=10 \mathrm{~nm}$. (d) Height histogram of the NPs obtained from (c). 

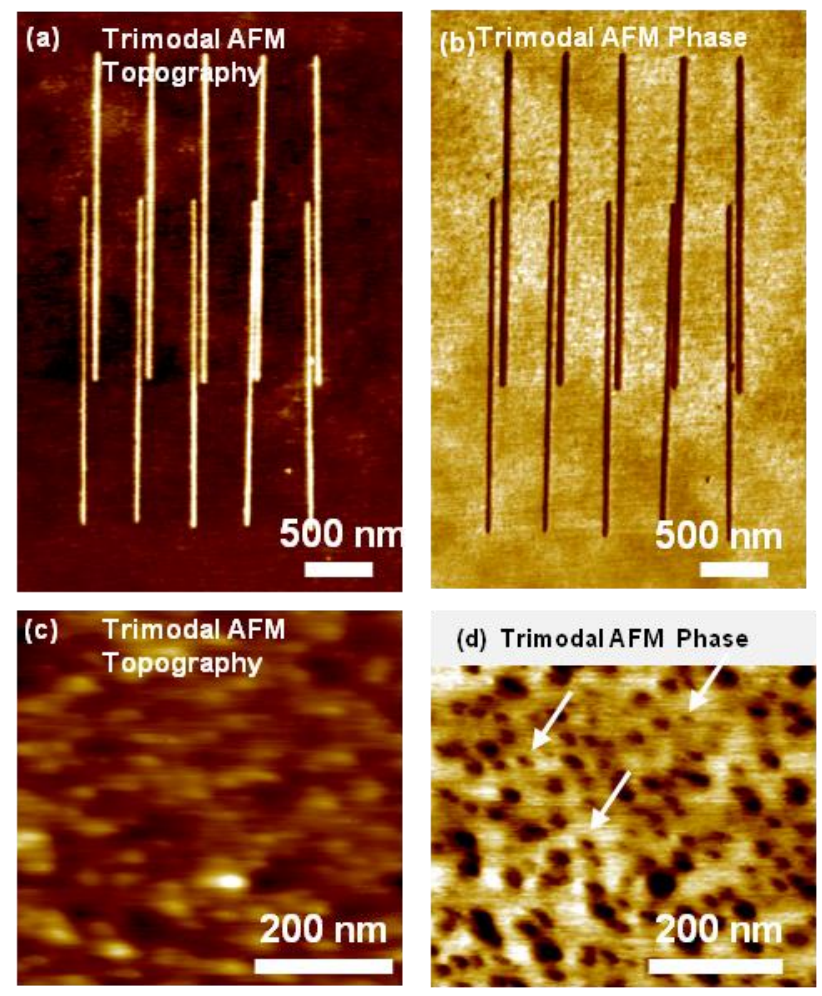

Figure 3. Trimodal AFM images of buried nanostructures. (a) Topography and (b) phase shift images of SiNWs buried under a $\sim 70 \mathrm{~nm}$ PDMS film. $A_{s p 1}=113 \mathrm{~nm} ; A_{0}=(130,2,12) \mathrm{nm}$ (c) Topography and (d) phase shift contrast of iron oxide NPs buried under a $\sim 65 \mathrm{~nm}$ PDMS film. The topography is acquired by keeping $A_{s p 1}=120 \mathrm{~nm} ; A_{0}=(130,1.5,40) \mathrm{nm}$ and the phase shift corresponds to $\phi_{2}$. The arrows indicate the position of NPs with a diameter below $9 \mathrm{~nm}$. 

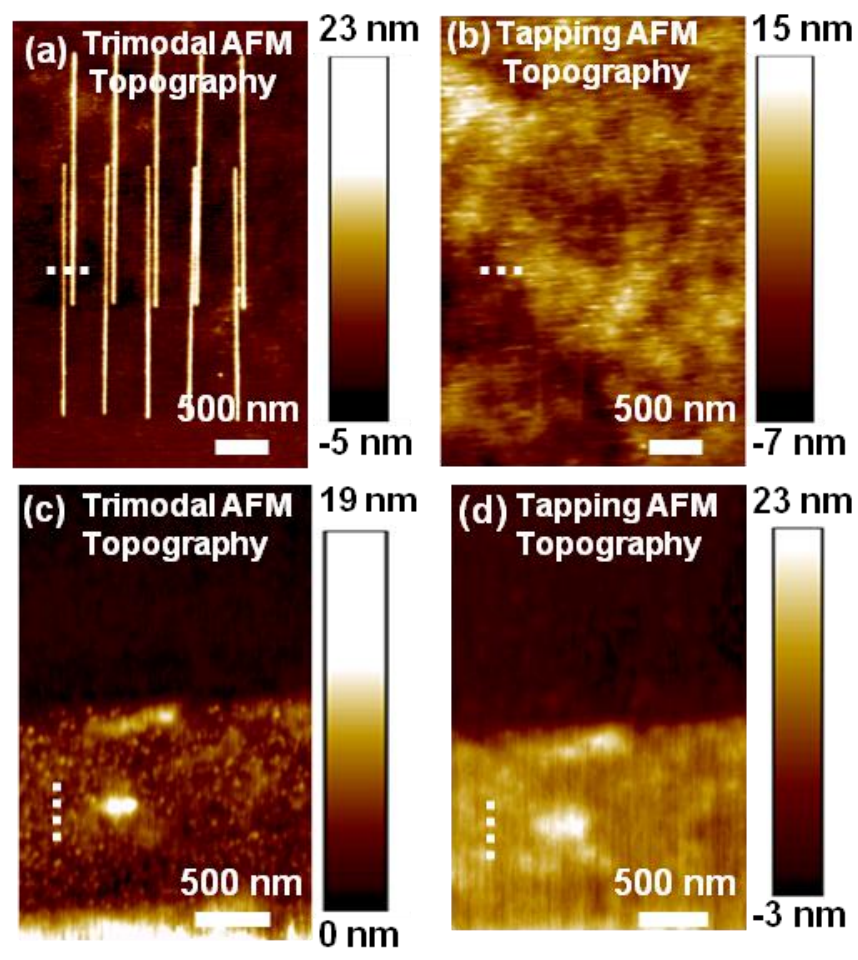

Figure 4. Comparison of tapping and trimodal AFM images. (a) Trimodal AFM image (topography) of an array of SiNWs buried under a $70 \mathrm{~nm}$ PDMS film; $A_{s p 1}=113 \mathrm{~nm} ; A_{0}=(130$, $1.5,40) \mathrm{nm}(\mathbf{b})$ Tapping mode AFM image of the same array; $A_{s p}=128 \mathrm{~nm} ; A_{0}=160 \mathrm{~nm}$. (c) Trimodal AFM image of a region that has a bare silicon surface and a section of iron oxide NPs coated by a $65 \mathrm{~nm}$ PDMS film. Under the PDMS there is a random distribution of NPs. $A_{s p 1}=100 \mathrm{~nm} ; A_{0}=(200,1.5,40) \mathrm{nm}$. (d) Tapping mode AFM image of the region shown in c. ; $A_{s p}=160 \mathrm{~nm} ; A_{0}=200 \mathrm{~nm}$. 

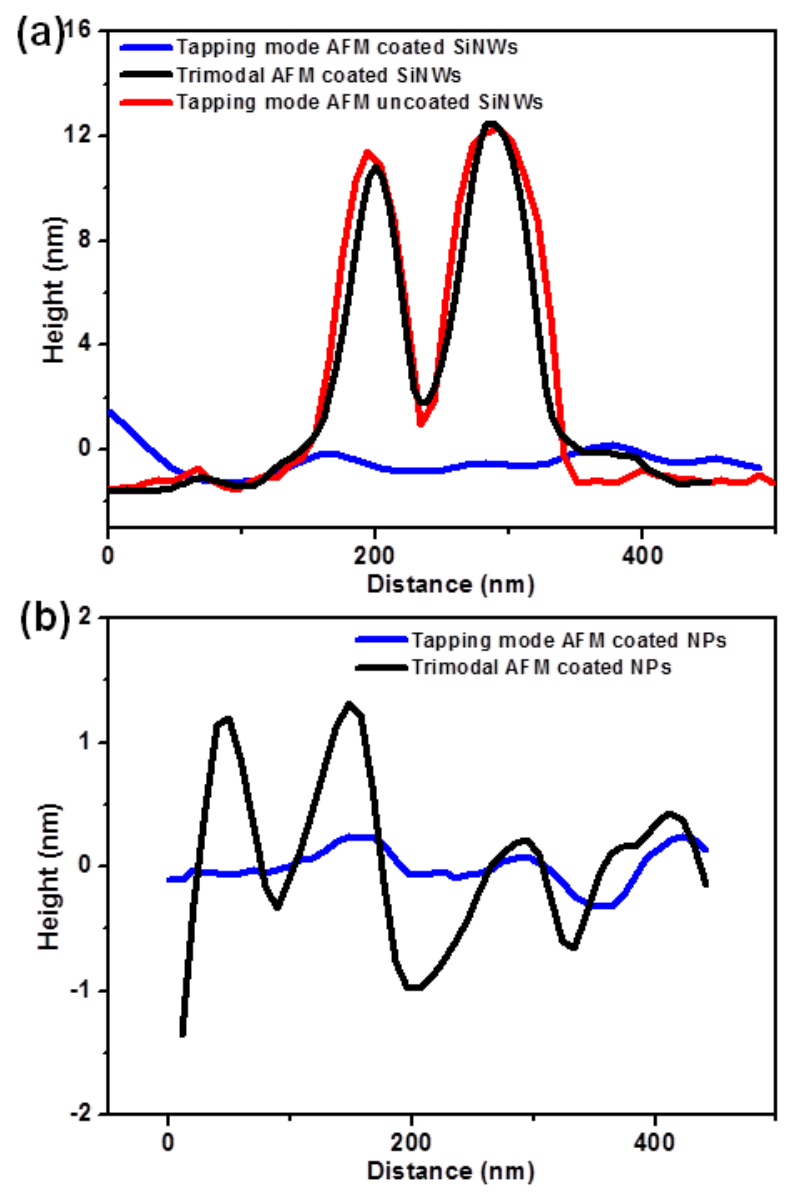

Figure 5. (a) Height cross-sections of the same section of a pair of SiNWs before and after the deposition of a PDMS film. The trimodal AFM profile matches the one obtained by tapping mode AFM before the deposition of PDMS. The tapping mode AFM image after PDMS deposition does not reveal the existence of SiNWs. (b) Height cross-section of NPs buried under PDMS; in black is the trimodal AFM cross-section; in blue is the tapping mode AFM crosssection. 

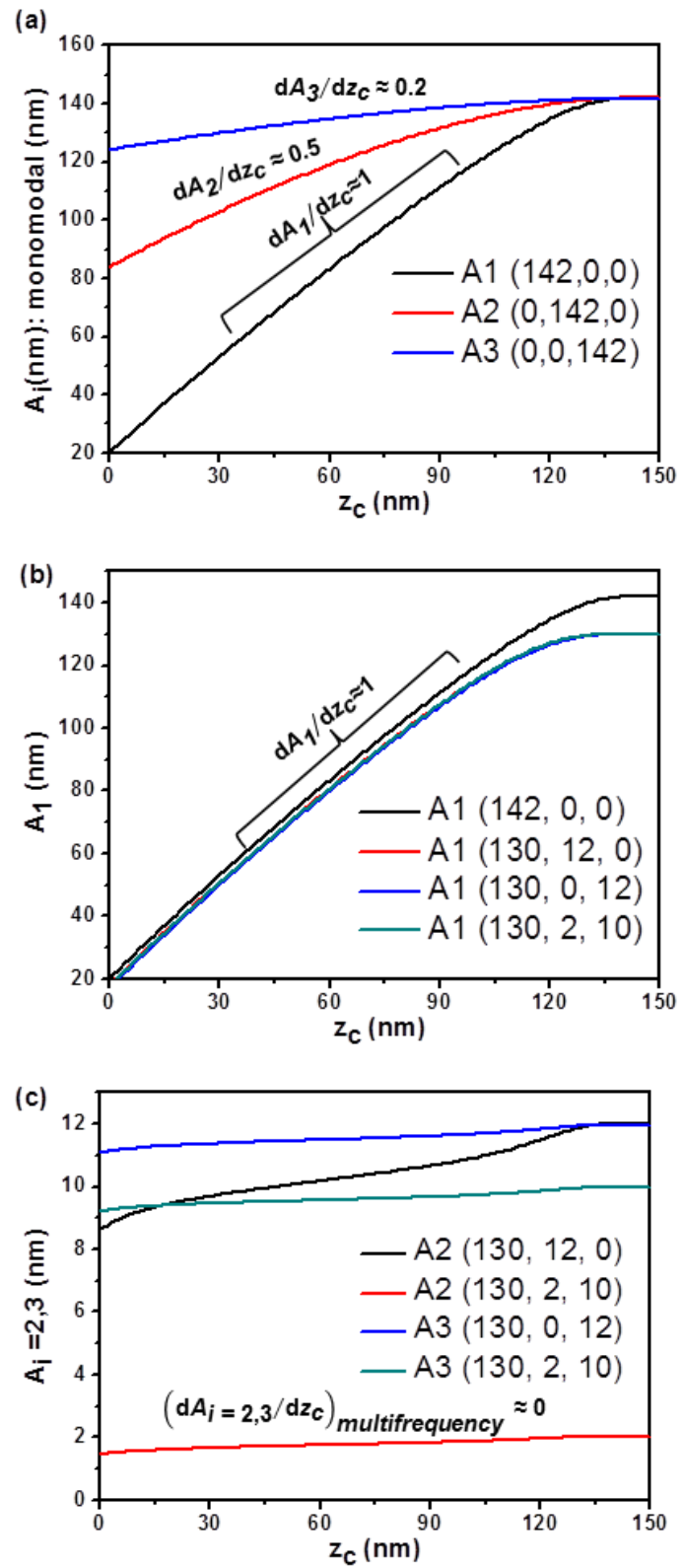

Figure 6. Dependence of the modal amplitude on the tip height distance $z_{c}$ (amplitude curve) for different dynamic AFM configurations. (a) Amplitude versus the $z_{c}$ for different monomodal excitations. The value of slope of the amplitude curves (central region) is also shown. (b) Amplitude curve of the first mode for monomodal and multifrequency configurations. (c) Amplitude curves for the second and third mode in multifrequency AFM. The slopes are nearly zero indicating that higher eigenmodes are unsuitable for feedback control in multifrequency AFM. The free oscillation amplitudes $\left(A_{01}, A_{02}, A_{03}\right)$ of the excited modes are expresses in $\mathrm{nm}$. 

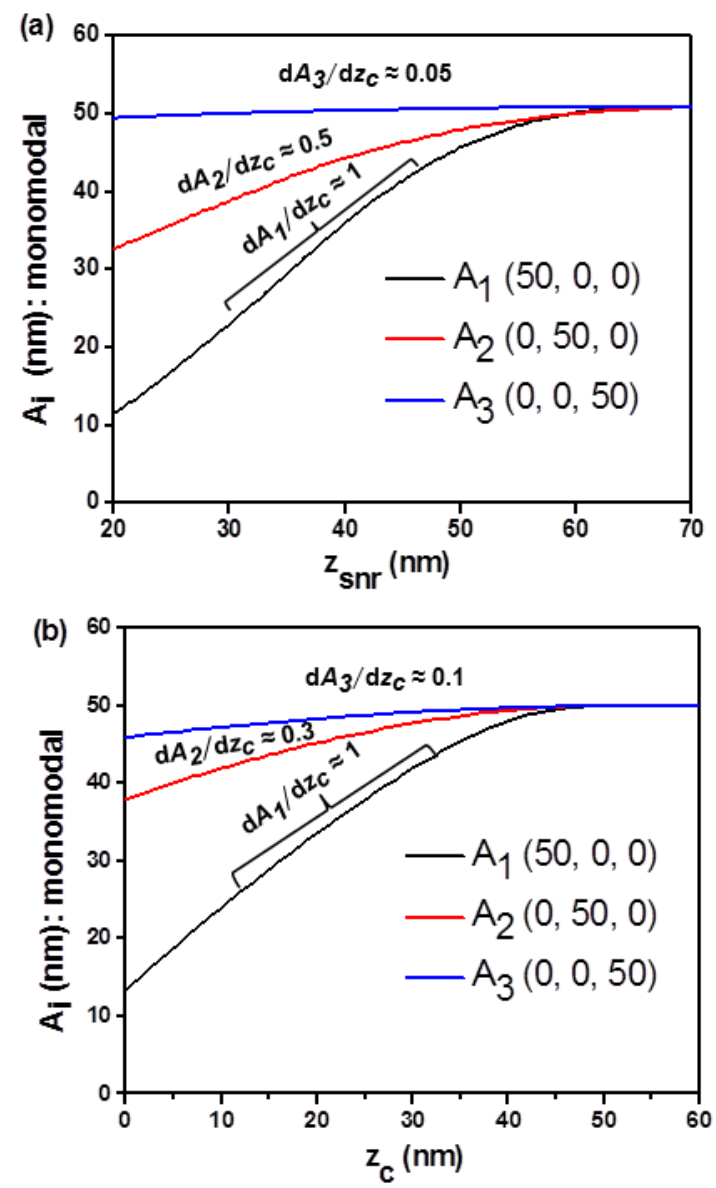

Figure 7. Experimental and numerical simulations amplitude curves. (a) Experimental amplitude curves for different monomodal excitations. (b) Numerical simulation amplitude curve for different monomodal excitations. The free oscillation amplitudes $\left(A_{01}, A_{02}, A_{03}\right)$ of the excited modes are expresses in nm. 

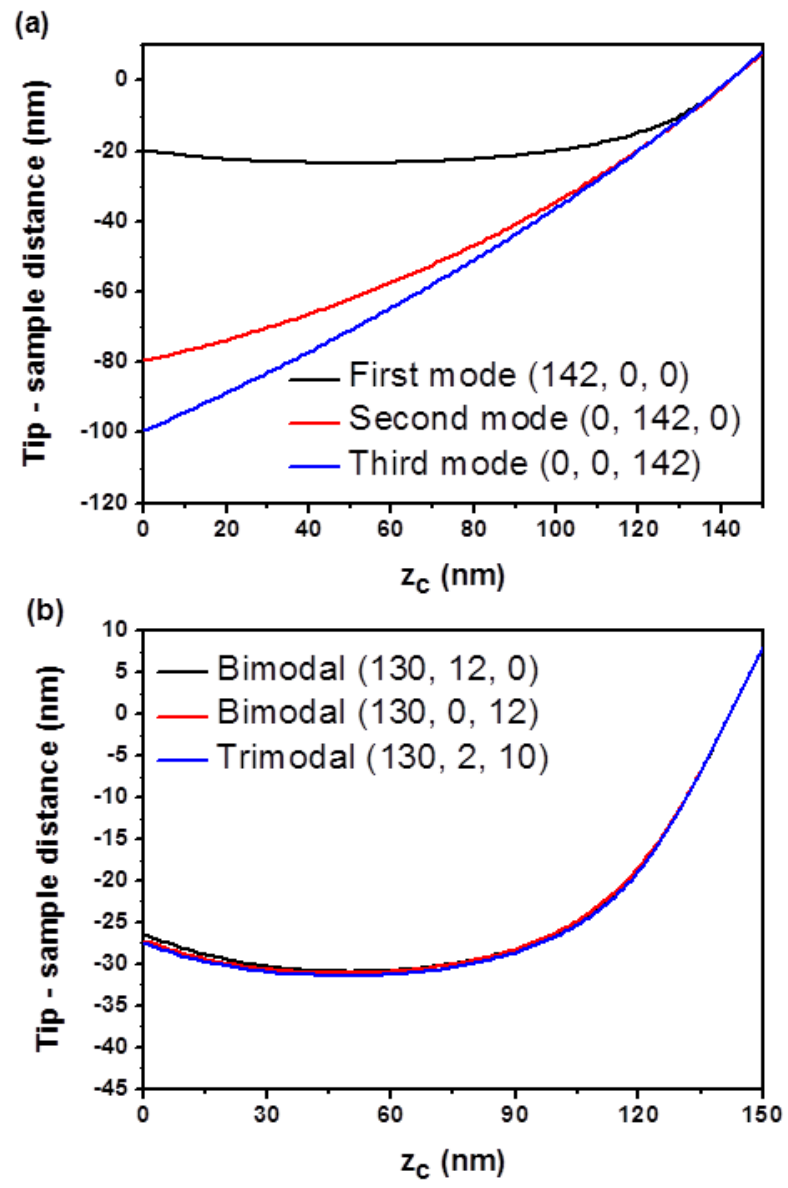

Figure 8. Tip penetration for different AFM configurations. (a) Tapping mode AFM. (b) Bimodal and trimodal AFM configurations. The free oscillation amplitudes of the excited modes $\left(A_{01}, A_{02}, A_{03}\right)$ are expressed in nm. 

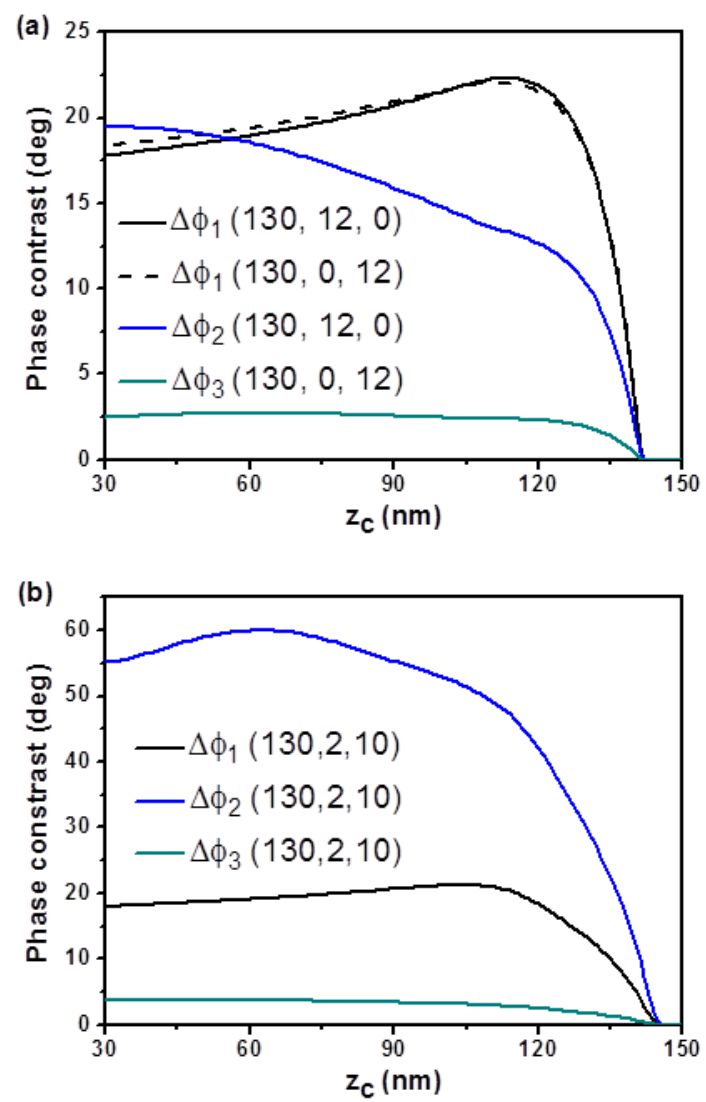

Figure 9. Subsurface phase shift contrast between a soft region (10 MPa) and relatively rigid region (1 GPa). (a) Bimodal AFM. (b) Trimodal AFM. The phase shift contrast is $\Delta \phi_{i}=\phi_{i}(10$ $\mathrm{MPa})-\phi_{i}(1 \mathrm{GPa})$. 


\section{REFERENCES}

[1] Raman A, Trigueros S, Cartagena A, Stevenson A P Z, Susilo M, Nauman E and Contera S A 2011 Mapping Nanomechanical Properties of Live Cells Using Multi-Harmonic Atomic Force Microscopy Nat. Nanotechnol. 6 809-814

[2] Goetz J G, Minguet S, Navarro-Lerida I, Lazcano J J, Samaniego R, Calvo E, Tello M, Osteso-Ibáñez T, Pellinen T, Echarri A, Cerezo A, Klein-Szanto A J P, Garcia R, Keely P J, Sanchez-Mateos P, Cukierman E and Del Pozo M A 2011 Biomechanical remodeling of the microenvironment by stromal caveolin-1 favors tumor invasion and metastasis Cell 146 148-163

[3] Porter A E, Gass M, Muller K, Skepper J N, Midgley P A and Welland M 2007 Direct imaging of single-walled carbon nanotubes in cells Nature Nanotechnology 2 713-717

[4] Alekseev A, Efimov A, Loos J, Matsko N and Syurik J 2014 Three-dimensional imaging of polymer materials by Scanning Probe Tomography Eur. Polym. J. 52 154-165

[5] Spitzner E C, Riesch C and Magerle R 2011 Subsurface Imaging of Soft Polymeric Materials with Nanoscale Resolution ACS Nano 5 315-320

[6] Shekhawat G, Srivastava A, Avashty S and Dravid V 2009 Ultrasound holography for noninvasive imaging of buried defects and interfaces for advanced interconnect architectures Appl. Phys. Lett 95263101

[7] Taubner T, Keilmann F and Hillenbrand R 2005 Nanoscale-resolved subsurface imaging by scattering-type near-field optical microscopy Optics Express 138893

[8] Cuberes M T, Assender H E, Briggs G A D and Kolosov O V 2000 Heterodyne force microscopy of PMMA/rubber nanocomposites: nanomapping of viscoelastic response at ultrasonic frequencies J. Phys. D 332347

[9] Shekhawat G S and Dravid V P 2005 Nanoscale imaging of buried structures via scanning near-filed ultrasound holography Science $\mathbf{3 1 0} 89-92$

[10] Tetard L, Passian A, Venmar K T, Lynch R M, Voy B H, Shekhawat G, Dravid V P and Thundat T 2008 Imaging nanoparticles in cells by nanomechanical holography Nat. Nanotechnol. 3501

[11] Tetard L, Passian A and Thundat T 2010 New modes for subsurface atomic force microscopy through nanomechanical coupling Nat. Nanotech. 5105

[12] Tetard L, Passian A, Lynch R M, Voy B H, Shekhawat G S, Dravid V P and Thundat T 2008 Elastic phase response of silica nanoparticles buried in soft matter Appl. Phys. Lett. 93 133113

[13] Verbiest G J, Simon J N, Oosterkamp T H and Rost M J 2012 Subsurface atomic force microscopy: towards a quantitative understanding Nanotechnology 23145704 
[14] Verbiest G J and Rost M J 2015 Beating beats mixing in heterodyne detection schemes Nat. Commun. 66444

[15] Garcia R 2010 Images from below the surface Nature Nanotech. 5 101-102

[16] Kimura K, Kobayashi K, Matsushige K and Yamada H 2013 Imaging of Au nanoparticles deeply buried in polymer matrix by various atomic force microscopy techniques Ultramicroscopy 133 41-49

[17] Vitry P, Bourillot E, Plassard C, Lacroute Y, Calkins E, Tetard L and Lesniewska E 2015 Mode - synthesizing atomic force microscopy for 3D reconstruction of embedded low-density dielectric nanostructures Nano Research 8 2199-2205

[18] Castaneda-Uribe O A, Riefenberger R, Raman A and Avila A 2015 Depth-sensitive subsurface imaging of polymer nanoscomposites using second harmonic Kelvin Probe Force Microscopy ACS Nano 9 2938-2947

[19] Jespersen T S and Nygard J 2007 3D Mapping of Individual Carbon Nanotubes Polymer/Nanotube Composites using Electrostatic Force Microscopy Appl. Phys. Lett. 90 183108

[20] Rawlings C, Wolf H, Hedrick J L, Coady D J, Duerig U and Knoll A W 2015 Accurate Location and Manipulation of Nanoescaled Objects Buried under Spin-Coated Films ACS Nano 9(6) 6188-6195

[21] Ebeling D, Eslami B and Solares S D 2013 Visualizing the Subsurface of Soft Matter: Simultaneous Topographical Imaging, Depth Modulation, and Compositional Mapping with Triple Frequency Atomic Force Microscopy ACS Nano 7 10387-10396

[22] Solares S D and Chawla G 2010 Triple-frequency intermittent contact atomic force microscopy characterization: Simultaneous topographical, phase, and frequency shift contrast in ambient air J. Appl. Phys. 108054901

[23] Solares S D and Chawla G 2010 Frequency response of higher cantilever eigenmodes in bimodal and trimodal tapping mode atomic force microscopy Meas. Sci. Technol. 21125502

[24] Garcia R and Proksch R 2013 Nanomechanical mapping of soft matter by modal atomic force microscopy European Polymer Journal 49 1897-1906

[25] Lozano J R and Garcia R 2009 Theory of phase spectroscopy in bimodal atomic force microscopy Phys. Rev. B 79014110

[26] Guzman H V, Garcia P D and Garcia R 2015 Dynamic force microscopy simulator (dForce): A tool for planning and understandind tapping mode and bimodal AFM experiments Beilstein J. Nanotech 6 369-379

[27] Garcia R and Herruzo E T 2012 The emergence of multifrequency AFM Nature Nanotechnol. 7 217-226 
[28] Solares S D 2014 Challenges and complexities of multifrequency atomic force microscopy in liquid environments Beilstein J. Nanotechnol. 5 298-307

[29] Rodriguez T R and Garcia R 2004 Compositional mapping of surfaces in atomic force microscopy by excitation of the second normal mode of the cantilever Appl. Phys. Lett. 84 449451

[30] Proksch R 2006 Multifrequency, repulsive - mode amplitude - modulated atomic force microscopy Appl. Phys. Lett. 89113121

[31] Eslami B, Ebeling D and Solares 2014 S D Trade-offs in sensitivity and sampling depth in bimodal atomic force microscopy and comparison to the trimodal case Beilstein J. Nanotechnol. 5 1144-1151

[32] Kawai S, Glatzel T, Koch S, Such B, Baratoff A and Meyer E 2009 Systematic achievement of improved atomic-scale contrast via bimodal dynamic force microscopy Phys. Rev. Lett. 103 220801

[33] Kawai S, Glatzel T, Koch S, Such B, Baratoff A and Meyer E 2010 Ultrasensitive detection of lateral atomic-scale interactions on graphite (0001) via bimodal dynamic force measurements Phys. Rev. B $\mathbf{8 1} 085420$

[34] Forchheimer D, Forchheimer R and Haviland D B 2015 Improving image contrast and material discrimination with nonlinear response in bimodal atomic force microscopy Nat. Comm. 66270

[35] Lai C Y, Barcons V, Santos S and Chiesa M 2015 Periodicity in bimodal atomic force microscopy J. Appl. Phys. 118044905

[36] Ang S M, Solares S D, Santos S and Ebeling P 2014 Energy transfer between eigenmodes in multimodal atomic force microscopy Nanotechnology $\mathbf{2 5} 47501$

[37] Herruzo E T, Perrino A P and Garcia R 2014 Fast nanomechanical spectroscopy of soft matter Nat. Commun. 53126

[38] Knoll A, Magerle R and Krausch G 2001 Tapping mode atomic force microscopy on polymers: Where is the true sample surface? Macromolecules 34 4159-4165

[39] Guzman H V, Perrino A P and Garcia R 2013 Peak Forces in High-Resolution Imaging of Soft Matter in Liquid ACS Nano 7 3198-3204

[40] Garcia R and San Paulo A 1999 Attractive and repulsive tip-sample interaction regimes in tapping-mode atomic force microscopy Phys. Rev. B 604961

[41] Garcia R, Martinez R V and Martinez J 2006 Nanochemistry and scanning probe nanolithographies. Chem. Soc. Rev 35 29-38

[42] Ryu Y K, Postigo P A, Garcia F and Garcia R 2014 Fabrication of sub-12 nm thick silicon nanowires by processing scanning probe lithography masks Appl. Phys. Lett. 104223112 
[43] Roca A G, Veintemillas-Verdaquer S, Port M, Robic C, Serna C J and Morales M P 2009 Effect of nanoparticle and aggregate size on the relaxometric properties of MR contrast agents based on high quality magnetite nanoparticles J. Phys. Chem. B 113 7033-7039

[44] Salas G, Casado C, Teran F J, Miranda R, Serna C J and Morales M P 2012 Controlled synthesis of uniform magnetite nanocrystals with high-quality properties for biomedical applications J. Mater.Chem. 2221065

[45] Garcia R 2010 Amplitude Modulation Atomic Force Microscopy Germany Wiley-VC Verlag GmbH \& Co. KGaA: Weinheim 104-105

[46] Martinez N F, Patil S, Lozano J R and Garcia R 2006 Enhanced compositional sensitivity in atomic force microscopy by the excitation of the first two flexural modes App. Phys. Lett. 89 153115

[47] Ramos J R, Pabijan J, Garcia R and Lekka M 2014 The softening of human bladder cancer cells happens at an early stage of the malignancy process Beilstein J. Nanotechnol. 5 447-457

[48] Roduit C, Sekatski S, Dietler G, Catsicas S, Lafont F and Kasas S 2009 Stifness tomography by atomic force microscopy Biophys. $J$ 97 674-677

[49] Dietz C, Herruzo E T, Lozano J R and Garcia R 2011 Nanomechanical coupling enables detection and imaging of $5 \mathrm{~nm}$ superparamagnetic particles in liquid Nanotechnology 22125708 